\title{
Efeito do Programa de Orientação a Pais no Desenvolvimento Lexical de Crianças Usuárias de Implante Coclear
}

\section{Effect of the Parents Guiding Program for the Lexical Development of Children using Cochlear Implant}

\author{
Juzzie de Melo Mota*, Talita Fortunato Tavares**, Robinson Tsuji Koji***, Ricardo Ferreira Bento****, \\ Carla Gentile Matas *****, Claudia Regina Furquim de Andrade******, Débora Befi Lopes *******. \\ *Fonoaudióga com Aprimoramento em Implante Coclear pelo HC/FMUSP \\ ** Doutoranda em Ciências da Reabilitação Departamento de Fisioterapia, Fonoaudiologia e Terapia Ocupacional da Faculdade de Medicina da Universidade de São Paulo. \\ *** Doutor em Otorrinolaringologia pela FMUSP \\ ***** Professor Titular da Faculdade de Medicina da Universidade de São Paulo. \\ ****** Professora Doutora da Faculdade de Medicina da Universidade de São Paulo. \\ ******* Professora Titular da Faculdade de Medicina da Universidade de São Paulo. \\ ******** Professora Associada da Faculdade de Medicina da Universidade de São Paulo \\ Instituição: Faculdade de Medicina da Universidade de São Paulo. \\ São Paulo/SP - Brasil. \\ Endereço para correspondência: Professora Associada Débora Befi-Lopes - Rua Cipotânea, 51 - Cidade Universitária - São Paulo / SP - Brasil - CEP: 05360-160 - Telefone: \\ (+55 11) 3091-8419 - Email: dmblopes@usp.br \\ Artigo recebido em 13 de Setembro de 2010. Artigo aprovado dem 7 de Outubro de 2010.
}

\section{RESUMO}

Introdução:

Crianças com perda auditiva profunda possuem atraso no repertório vocabular em comparação a crianças ouvintes de mesma idade cronológica. Um dos fatores considerados fundamental durante o processo de desenvolvimento da linguagem é a intervenção da família.

Objetivo:

Propor um programa controlado para orientação a pais de crianças usuárias de implante coclear (CIC) e verificar a efetividade do programa através da variação de desempenho lexical das crianças.

Método:

Estudo prospectivo.Participaram da pesquisa oito CIC e suas respectivas famílias as quais foram divididas em dois grupos: GI (com programa de orientação) e GII (sem programa de orientação). O desempenho lexical das crianças foi mensurado através do LAVE (Lista de Avaliação do Vocabulário Expressivo) pré e pós-programa de orientação. O programa de orientação Família Participativa para CIC foi constituído por quatro sessões, de aplicação semanal, com duração de 60 minutos cada. Os temas abordados foram desenvolvimento de habilidades auditivas e linguísticas.

Resultados: As ANOVAs intra-grupos não indicaram desempenho significantemente diferenciado no LAVE na comparação pré- e pós programa de orientação em ambos os grupo. ANOVAs entre-grupos não revelaram diferença estatisticamente significante entre os grupos para performance lexical.

Conclusão: Os grupos não se diferenciaram na efetividade do programa como medido pelo LAVE. Tal resultado deve ser um indicador importante de medidas de evolução lexical baseadas somente na percepção familiar. Em estudos futuros devem ser considerados extensão temporal do programa, expansão da amostra, e medidas objetivas do desenvolvimento lexical.

Palaviras-chave: implante coclear, desenvolvimento da linguagem, linguagem, linguagem infantil.

\section{SUMMARY}

Introduction:

Children with severe hearing loss have a delay in the vocabulary repertoire compared to the normal-hearing children of the same chronological age. One of the factors considered to be basic during the process of language development is the family intervention.

Objective: To propose a program controlled for guiding of parents of children using cochlear implant (CIC) and verify the effectiveness of the program through the variation of the children's lexical performance.

Method: Prospective study. Eight CIC and their respective families took part in the research and were divided into two groups: GI (with guiding program) and GII (without guiding program). The lexical performance of the children was measured through the List of Evaluation of Expressive Vocabulary (LAVE) with prior and posterior guiding program. The guiding program Participative Family for CIC was composed by four sessions, of weekly application, with duration of 60 minutes each. The topics approached were development of auditory and linguistic skills.

Results: The intra-groups ANOVAs didn't present a different significant performance in LAVE for the prior and posterior guiding comparison of both groups. The ANOVAs between groups didn't reveal a statistically significant difference between the groups for lexical performance.

Conclusion: The groups didn't differ in the effectiveness of the program as measured by LAVE. Such results must be an important indicator of lexical evolution measures based only on the family perception. In future studies the program temporal extension, sample expansion and objective measures of the lexical development must be taken into account. 


\section{INTRODUÇÃO}

Odesenvolvimento lexical relaciona-se de maneira direta a o desenvolvimento da linguagem. O desempenho em testes padronizados de vocabulário tem sido utilizado em muitos estudos como preditor de atrasos linguísticos tanto de crianças ouvintes quanto de crianças com deficiências auditivas usuárias de aparelhos de amplificação sonora individuais (AASI) e implante cocleares (IC) $(1,2,3,4,5,6)$.

O MacArthur-Bates Communicative Development $(7,8,9)$ e o Language Development Survey (LDS) $(10,11)$ são instrumentos bastante utilizados para verificar o desenvolvimento do lexical. Ambos os testes são compostos por uma checklist de palavras a qual deve ser preenchida pelos pais. Validações concordantes entre os dois testes já foram comprovadas e são amplamente aceitas na literatura $(11,12)$. Uma versão e adaptação do LDS para o Português Brasileiro foi publicada por Capovilla (13) sendo o nome do teste traduzido para Lista de Avaliação de Vocabulário Expressivo (LAVE). Até o momento, não são reportadas pesquisas com estes instrumentos em crianças brasileiras usuárias de IC.

Pesquisas revelam que crianças com perda auditiva profunda possuem atraso no repertório vocabular em comparação a ouvintes de mesma idade cronológica $(3,14,15,16)$. Um dos fatores considerados responsáveis é o fato de a integridade anátomo-fisiológica do sistema auditivo constitui um pré-requisito para a aquisição e o desenvolvimento normal das habilidades linguísticas acarretando, portanto, em déficit no desenvolvimento lexical. Outro fator fundamental durante o processo de desenvolvimento da linguagem é a intervenção da família. O desempenho de linguagem de crianças é influenciado positivamente pelo envolvimento familiar e interação mãe-filho, e consequentemente, pelo input linguístico dado pela família o qual determinará o léxico da criança.
Com base nos programas de reabilitação com família participativa - prática baseada em evidências - o objetivo da pesquisa realizada foi à proposição de um programa controlado para a orientação a pais de crianças surdas usuárias de implante coclear. O programa recebeu o título de Familia Participativa para Crianças Surdas Usuárias de Implante Coclear. Este programa abordou temáticas relacionadas ao desenvolvimento das habilidades linguísticas e auditivas. A hipótese do estudo é a verificação da efetividade do programa pela variação de desempenho das crianças, pré e pós-programa, pelo teste LAVE.

\section{MÉTOdo}

Esta pesquisa foi aprovada pela comissão de ética da instituição sob protocolo número 0290/10. Todos os responsáveis assinaram termo de consentimento livre e esclarecido concordando com a participação na pesquisa e na posterior divulgação dos dados. Seguindo os preceitos éticos e morais pertinentes os familiares das crianças em grupo controle estavam cientes que no caso de confirmação da hipótese do estudo haveria o imediato início de programa de orientação específico para esse grupo.

\section{Casuística}

Participaram da pesquisa oito crianças surdas usuárias de implante coclear (CIC) atendidas no Ambulatório de Otorrinolaringologia do Hospital das Clínicas da Faculdade de Medicina da Universidade de São Paulo (HCFMUSP) e suas respectivas famílias (Tabela 1).

A seleção inicial das famílias foi realizada com base no questionário econômico Critério de Classificação Econômica Brasil(17) (CCEB). Este questionário classificou as famílias de classes econômicas e graus de escolaridade semelhantes.

Tabela I. Caracterização dos sujeitos de acordo com o grupo (Gl e GII).

\begin{tabular}{|c|c|c|c|c|c|c|}
\hline Grupo & Sujeito & $\begin{array}{c}\text { Idade } \\
\text { cronológica } \\
\text { (meses) }\end{array}$ & $\begin{array}{l}\text { Idade } \\
\text { auditiva } \\
\text { (meses) }\end{array}$ & $\begin{array}{l}\text { Classificação } \\
\text { econômica* }\end{array}$ & $\begin{array}{l}\text { Nívelde } \\
\text { escolaridade do } \\
\text { chefe dafamília }\end{array}$ & $\begin{array}{l}\text { Permeabilidade da } \\
\text { família - PM (\%) }\end{array}$ \\
\hline$\overline{G l}$ & I & 97 & 28 & $\mathrm{C} 2$ & Fundamentalll incompleto & 47,5 \\
\hline $\mathrm{Gl}$ & 2 & 82 & 34 & B2 & Médio completo & 90 \\
\hline Gl & 3 & 64 & 18 & $\mathrm{Cl}$ & Médio completo & 95 \\
\hline Gl & 4 & 78 & 9 & $\mathrm{C} 2$ & Fundamental completo & 80 \\
\hline Gll & 5 & 68 & 29 & B2 & Fundamentall completo & 42,5 \\
\hline Gll & 6 & 120 & 42 & B2 & Médio completo & 92,5 \\
\hline Gll & 7 & 70 & 15 & $\mathrm{C} 2$ & Superiorincompleto & 95 \\
\hline Gll & 8 & 67 & 15 & $\mathrm{Cl}$ & Fundamentalll incompleto & 47,5 \\
\hline
\end{tabular}

Legenda: *Critério CCEB (2008): A1 renda de 9.733,47reais, A2 de 6.563,73, B1 de 3.479,36, B2 de 2.012,67, C1 de 1.194,53, C2 de $726,26, \mathrm{D}$ de 484,97 e E de 276,70 reais. 
Os participantes foram divididos em dois grupos:

- Grupo Pesquisa (GI): quatro CIC e seus respectivos pais, participantes do programa de orientação- Familia Participativa CIC;

- Grupo Controle (GII): quatro CIC e seus respectivos pais, não participantes do programa de orientação.

A escolha para composição de GI e GII foi realizada por meio do uso de números aleatórios (Extrato de Tábula de Números Aleatórios). O pareamento dos grupos foi realizado com base no questionário de Permeabilidade da Familia que possibilita qualificar a abertura da família quanto ao processo terapêutico (18). O critério de pareamento foi por pontuação similar ou de variação inferior a 1 .

\section{Procedimentos}

Etapa 1: Composição dos Grupos

Os familiares (pai e/ou mãe) foram convidados a disponibilizar voluntariamente seus dados socioeconômicos para a pesquisa. Após concordarem, familiares foram convocados individualmente em uma sala separada para preenchimento do questionário. Durante o preenchimento do questionário, a pesquisadora apresentou as perguntas oralmente aos participantes, transcrevendo suas respostas. O tempo utilizado no preenchimento variou de cinco a dez minutos por participante. Uma vez compatibilizados os extratos econômicos e níveis de escolaridade foram sorteados e pareados os grupos.

\section{Etapa 2: Aplicação do LAVE}

OLAVE (teste de passa/falha onde os pais assinalam as palavras que são faladas, espontaneamente, pelas crianças, nas diversas categorias lexicais) foi aplicado em duas coletas. O pré-teste foi aplicado em GI e GII no mesmo período de tempo. O pós-teste de GI foi aplicado imediatamente após o fim do programa para todos os participantes (entre 30 e 40 dias). O pós-teste de GII foi aplicado entre 30 e 40 dias após o pré-teste para todos os participantes.

Etapa 3: Realização do Programa de Família Participativa para Crianças Surdas Usuárias de Implante Coclear.

O Programa de Familia Participativa para Crianças Surdas Usuárias de Implante Coclear foi constituído por quatro sessões, de aplicação semanal, com duração de 60 minutos cada. A duração do programa foi de quatro semanas. Os módulos abordados foram: habilidades auditivas e habilidades linguísticas. As sessões foram estruturadas na forma: objetivo; conteúdo programático; técnica de abordagem e relação dialética (19).

\section{RESULTADOS}

\section{Análise intra-grupos}

A análise intra-grupos foi realizada considerando a predominância entre as categorias lexicais no pré, pós e na comparação pré-pós aplicação do LAVE. Foram utilizados os testes de Kruskal-Wallis ANOVA por ranques nas testagens pontuais e Friedman ANOVA para a testagem comparativa. As Figuras 1 e 2 permitem visualização desses resultados.

Para 9GI foi obtido:

- Pré-teste - H (13, N=42)=22.007 p = .055; indicando uma tendência a desempenho diferenciado entre as categorias;

- Pós-teste - H (13, N=42)=15.621 p = .270; indicando que não há desempenho diferenciado entre as categorias;

- Pré x Pós $-(\mathrm{N}=42, \mathrm{gl}=1)=9.783 \mathrm{p}=.002^{*}$; indicando que há desempenho significantemente diferenciado entre as performances pré e pós.

Para GII foi obtido:

- Pré-teste - H (13, N=42)=30.764 p = .003*; indicando uma tendência a desempenho diferenciado entre as categorias;

- Pós-teste - H (13, N=42)=15.459 p = .280; indicando que não há desempenho diferenciado entre as categorias;

- Pré x Pós $-(\mathrm{N}=42, \mathrm{gl}=1)=2.454 \mathrm{p}=.117$; indicando que não há desempenho significantemente diferenciado entre as performances pré e pós.

\section{Análise entre-grupos}

A análise entre-grupos foi realizada considerando a performance entre os grupos no pré-teste, no pós-teste e quanto à evolução lexical dos grupos. O teste utilizado foi ANOVA de um fator. A Tabela 2 e a Figura 3 permitem a visualização dessa comparação.

Comparação no Pré-teste- $\mathrm{F}(1,82)=1.9568, \mathrm{p}=.1656$, indicando que não há diferença significante entre os grupos.

Comparação no Pós-teste - $\mathrm{F}(1,82)=.0006, \mathrm{p}=.979$, indicando que não há diferença significante entre os grupos. 


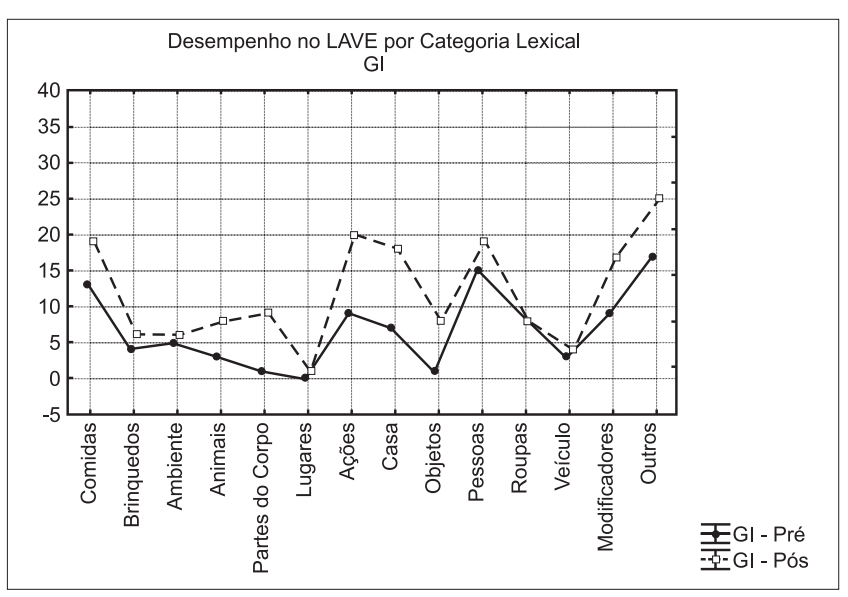

Figura 1. Desempenho no LAVE por categoria lexical - GI.

Tabela 2. Comparação entre grupos no Pré x Pós-teste.

\begin{tabular}{lcc}
\hline \multicolumn{3}{c}{ Comparação entre grupos das diferenças de pontuação prée és } \\
\hline Grupol & Média & Desvio Padrão \\
Grupoll & I I & 15,52 \\
\hline
\end{tabular}

Comparação Pré x Pós-teste-Apesar de, em média, a evolução no desempenho lexical ter sido maior para o GI, não é observada diferença estatisticamente significante entre os dois grupos através de ANOVA de um fator.

\section{DISCUSSÃO}

As análises quantitativas do estudo indicam não haver influência do Familia Participativa CIC na expansão do vocabulário quando usado o teste LAVE (percepção familiar).

Numa análise qualitativa pode ser observado que para o Grupo I a média de palavras adquiridas foi maior que para GII. Para cada palavra adquirida pelo Grupo II, 2,18 palavras foram adquiridas pelo Grupo I indicando uma influência positiva da aplicação do programa de Familia Participativa CIC na expansão do vocabulário das crianças.

Outro dado de natureza qualitativa foi observado durante a aplicação do programa - o aprendizado das famílias em relação às habilidades linguísticas e auditivas de suas crianças.

Odelineamento da pesquisa pode ser caracterizado como experimental e os dados obtidos considerados um estudo piloto sobre o tema. Na expansão do estudo



Figura 2. Desempenho no LAVE por categoria lexical - GII

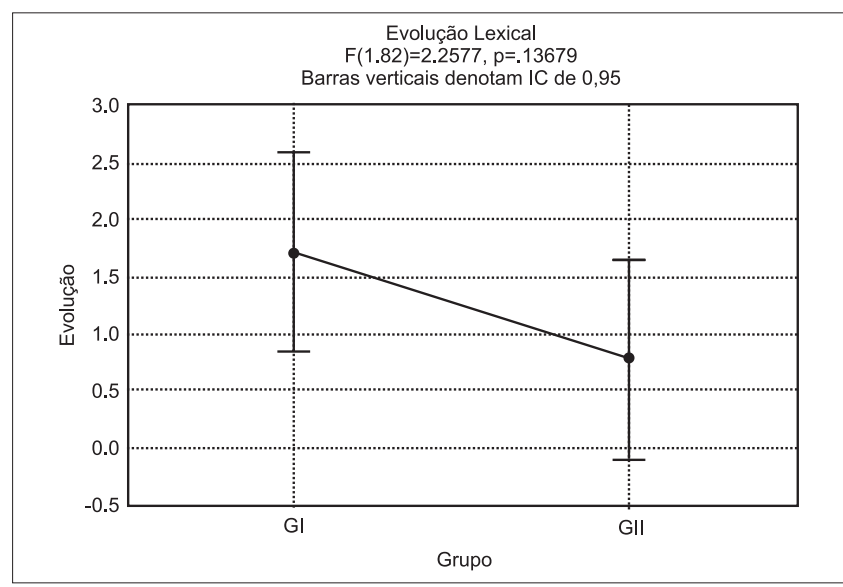

Figura 3. Evolução Lexical de ambos os grupos (GI e GII).

deverão ser considerados tanto a extensão do programa quanto à introdução de um instrumental objetivo de avaliação do desenvolvimento do léxico das crianças. O número de participantes, embora seja compatível com estudos semelhantes, é uma variável de importância e deve ser expandido.

Apesar das limitações do estudo esta pesquisa é de interesse frente ao limitado referencial bibliográfico sobre o tema - desenvolvimento da linguagem em crianças brasileiras usuárias de Implante Coclear. Com a evolução dos estudos brasileiros nessa área serão possíveis às comparações cross-languagee assim o conhecimento sobre a real influência da língua no desenvolvimento da linguagem nas crianças usuárias do implante coclear.

\section{CONCLUSÃO}

A hipótese do estudo não foi confirmada. Os grupos não se diferenciaram na verificação da efetividade do 
programa pela variação de desempenho das crianças, pré e pós-programa, pelo teste LAVE. Esse resultado deve ser considerado um indicador importante sobre as avaliações de evolução de vocabulário baseadas exclusivamente na percepção familiar. As principais variáveis que devem ser consideradas nos estudos futuros são: expansão da amostra; testagem objetiva com instrumento de avaliação de vocabulário validado; extensão temporal do programa.

\section{REFERÊNCIAS BIBLIOGRÁFICAS}

1. Torres MLGM, Maia HA, Perissinoto J, Ferreira VJA. Descrição do Léxico Expressivo de Crianças aos 5 anos de idade. Rev Cefac. 2002, 4:241-251.

2. Nott P, Cowan R, Brown M, Wigglesworth G. Early Language Development in Children with Profound Hearing Loss Fitted with a Device at a Young Age: Part I-The Time Period Taken to Acquire First Words and First Word Combinations. Ear \& Hearing. 2009, 30(5):526-540.

3. Nott P, Cowan R, Brown M, Wigglesworth G. Early Language Development in Children with Profound Hearing Loss Fitted with a Device at a Young Age: Part II-Content of the First Lexicon. Ear \& Hearing. 2009, 30(5):541-551.

4. Schorr EA, Roth FP, Fox NA. A Comparison of the Speech and Language Skills of Children With Cochlear Implants and Children With Normal Hearing. Communication Disorders Quarterly. 2008, 29(4):195-210.

5. Ertmer DJ, Strong LM, Sadagopan N. Beginning to Communicate After Cochlear Implantation: Oral Language Development in a Young Child. Journal of Speech, Language \& Hearing Research. 2003, 46(2):328.

6. Miyamoto RT, Hay-Mccutcheon MJ, Kirk KI, Houston DM, Bergeson-Dana T. Language skills of profoundly deaf children who received cochlear implants under 12 months of age: a preliminary study. Acta Oto-Laryngologica. 2008, 128(4):373-377.

7. Geers AE, Nicholas JG. Expected Test Scores for Preschoolers With a Cochlear Implant Who Use Spoken Language. American Journal of Speech-Language Pathology. 2008, 17:121-138.

8. Thal D, DesJardin JL, Eisenberg LS. Validity of theMacArthur-BatesCommunicative Development Inventories for Measuring Language Abilities in Children With Cochlear Implants. American Journal of Speech-Language Pathology. 2007, 16:54-64.

9. Rinaldi P, Caselli C. Lexical and Grammatical Abilities in
Deaf Italian Preschoolers: The Role of Duration of Formal Language Experience. Journal of Deaf Studies and Deaf Education. 2009, 14(1):63-75.

10. Rescorla L, Achenbach T. Use of the Language Development Survey (LDS) in a National Probability Sample of Children 18 to 35 Months Old. Journal of Speech, Language \& Hearing Research. 2002, 45(4):733.

11. Rescorla L, Alley A. Validation of the Language Development Survey (LDS): A Parent Report Tool for Identifying Language Delay in Toddlers. Journal of Speech, Language \& Hearing Research. 2001, 44(2):434.

12. Rescorla L, Ratner N, Jusczyk P, Jusczyk A. Concurrent Validity of the Language Development Survey: Associations With the MacArthur-Bates Communicative Development Inventories: Words and Sentences. American Journal of Speech-Language Pathology. 2005, 14(2):156-163.

13. Capovilla FC, Capovilla AGS. Desenvolvimento Linguístico na Criança dos Dois aos Seis Anos: Tradução e Estandardização do Peabody Picture Vocabulary Test de Dunn \& Dunn, e da Language Development Survey de Rescorla. Ciência Cognitiva: Teoria, Pesquisa e Aplicação. 1997, 1(1):353-380.

14. Kiese-Himmel C, Reeh M. Assessment of expressive vocabulary outcomes in hearing-impaired children with hearing aids: do bilaterally hearing-impaired children catch up? The Journal of Laryngology \& Otology . 2006, 120:619626.

15. Melo TM, Moret ALM, Bevilacqua MC. Avaliação da produção de fala em crianças deficientes auditivas usuárias de Implante Coclear Multicanal. Rev Soc Bras Fonoaudiol. 2008, 13(1):45-51.

16. DesJardin JL, Eisenberg LS. Maternal Contributions: Supporting Language Development in Young Children with Cochlear Implants. Ear \& Hearing, 28(4):456-469.

17. ABEP - Associação Brasileira de Empresas de Pesquisa (2008). [homepage da Internet]. Acesso em 02 de julho de 2009. Disponível em: www.abep.org.

18. Bevilacqua MC, Yamada MO, Moret ALM. Critérios de Referência quanto ao Grau de Permeabilidade da Família noProcesso Terapêutico. Centro de Pesquisas Audiológicas, Hospital de Reabilitação de Anomalias Craniofaciais, Universidade de São Paulo - CPA/ HRAC-USP, Agosto, 2001.

19. Takahashi RT, Fernandes MFP. Plano de aula: conceitos e metodologia. Acta Paul. Enf. 2004, 17(1):114-118. 